\title{
Sneutrino Condensate Source for Density Perturbations, Leptogenesis, and Low Reheat Temperature
}

\author{
Anupam Mazumdar ${ }^{1}$ and Abdel Pérez-Lorenzana ${ }^{2}$ \\ ${ }^{1}$ CHEP, McGill University, 3600 University Road, Montréal, Québec, H3A 2T8, Canada \\ ${ }^{2}$ Departamento de Física, Centro de Investigación y de Estudios Avanzados del I.P.N., \\ Apartado Post. 14-740, 07000, México, Distrito Federal, México
}

(Received 22 November 2003; published 24 June 2004)

\begin{abstract}
We bring together some known ingredients beyond the standard model physics that can explain the hot big bang model with the observed baryon asymmetry and also the fluctuations in the cosmic microwave background radiation with a minimal set of assumptions. We propose an interesting scenario where the inflaton energy density is dumped into an infinitely large extra dimension. Instead of the inflaton it is the right handed sneutrino condensate, which is acquiring a nonzero vacuum expectation value during inflation, whose fluctuations are responsible for the density perturbations seen in the cosmic microwave background radiation with a spectral index $n_{s} \approx 1$. The decay of the condensate is explaining the reheating of the Universe with a temperature, $T_{r h} \leq 10^{9} \mathrm{GeV}$, and the baryon asymmetry of order one part in $10^{10}$ with no baryon-isocurvature fluctuations.
\end{abstract}

DOI: 10.1103/PhysRevLett.92.251301

PACS numbers: 98.80.Cq, 11.25.Wx, 12.60.Jv

Inflation is the most successful paradigm that can explain the scale invariant density perturbations [1], the horizon, and flatness problems; however, inflation alone cannot explain the observable Universe, because inflation leaves the Universe cold and devoid of (almost) any entropy. It is usually believed that after inflation the inflaton energy density will be released into the observable world with the standard model (SM) degrees of freedom. This last but essential point has often been sidelined in many discussions. The success of inflation lies only if it facilitates the big bang nucleosynthesis [2].

There are many models of inflation that claim to be satisfying all the criteria [3], in spite of the fact that the inflaton's identity is largely unknown, and often in the literature it is regarded as a gauge singlet. Recently with an advent of stringy motivated inflation, it is possible to realize accelerated expansion by a geometric stretching of the space time [4], by inducing potentials from slowly moving D-branes [5], or via multiple tachyon condensations [6].

In all these cases the Universe is assumed to be a fourdimensional surface (brane) embedded in a bulk of $4+n$ dimensions. The $n$ extra dimensions can be either open [7] or compact [8] (for a review see Ref. [9]). Usually in the brane world models, the SM fields can be considered to be four dimensional and localized at the brane, whereas inflation could be driven by either bulk or brane fields [10], or due to the modification of the bulk space time geometry, as it is in the anti-de Sitter (AdS) case [11]. All these suggest that there is no dearth of inflationary models (for a review on string inspired cosmology, see [12]).

The main aim of this Letter is to illustrate a scenario within the brane world setup where after inflation the brane is devoid of the inflaton energy density, and therefore the observable world is virtually cold. In our picture, inflaton plays no role in postinflationary cosmology. It is rather the sneutrino condensate that does the whole job of reheating our Universe, generating primordial density perturbations and providing baryogenesis via leptogenesis.

Such a scenario is possible if the brane inflaton couples in such a way that it preferably decays into the bulk degrees of freedom, which could be either fermions, scalars, or gravitons. This could happen, for instance, if the inflaton carries some global quantum numbers not being carried by the brane degrees of freedom, but by some bulk fields. In this case inflaton energy density cannot be dumped into the SM fields living on the brane.

For simplicity we work on the 5D setup, where the bulk is infinitely large with a warped metric: $d s^{2}=$ $e^{-k|z|} g_{\mu \nu} d x^{\mu} d x^{\nu}-d z^{2}$, with all the SM fields attached to the brane [7]. Here $k$ is a constant that relates the fundamental five-dimensional scale of gravity, $M_{5}$, with Planck scale $M_{P} \sim 2.4 \times 10^{18}$, by $k M_{P}^{2}=M_{5}^{3}$. We assume that $k$ is close to the Planck scale to facilitate the standard Hubble expansion on the brane [13].

In such a background the wave functions of any bulk fields have a continuum spectrum $[9,14]$ that starts at $m=0$, meaning that its Kaluza-Klein (KK) modes can take any momentum along the extra space coordinate. Now let us assume that the inflaton, which is a brane scalar field, couples to the bulk degrees of freedom. In this case the inflaton can decay into all the continuum KK modes below its mass, therefore draining its energy from the brane and into the bulk. Note that the inflaton energy density is gradually redshifted into the bulk before becoming vanishingly small at the brane; this is due to the fact that the fifth dimension is warped such that the released energy density into the bulk appears to be redshifted from the brane observer $[7,9,14]$. 
Our scenario can be thought of as a hot radiating plate cooling down by emitting its energy into a cold surrounding. It is not hard to see that this cooling process is extremely efficient. Let us consider, for instance, the coupling, $\phi \bar{\psi} \psi \delta(y) / M_{5}$, where $\phi$ is the inflaton of mass $m_{\phi}$, and $\psi$ is a bulk fermion in an AdS background. The typical KK expansion of the wave function of the bulk field goes as $\psi(x, z)=\int(d m / k) \psi_{m}(x) h_{m}(z)$, where at the brane position $h_{m}(0) \sim \sqrt{m}$, with $m$ being the KK mass [14]. The total decay rate of the inflaton into KK modes is given by

$$
\begin{aligned}
\Gamma & =\int_{0}^{m_{\phi}} d m d m^{\prime}\left(m_{\phi} / k^{2} M_{5}^{2}\right)\left|h_{m}(0) h_{m}^{\prime}(0)\right|^{2}, \\
& \approx\left(m_{\phi} / M_{5}\right)^{4}\left(M_{P} / M_{5}\right)^{4} m_{\phi} .
\end{aligned}
$$

Often the inflaton is typically heavy, say, about the grand unified theory (GUT) scale. Let us assume $M_{5} \sim 0.1 M_{p}$; then we find the inflaton decay rate is quite fast compared to the Hubble expansion rate after the end of inflation, e.g., $H \sim m_{\phi}$.

Note, however, that these bulk fields may not be completely harmless. As they move towards the fifth dimension, their energy density might eventually collapse to form a black hole at the AdS horizon [15]. The presence of a black hole in the bulk induces a contribution to the brane expansion that looks like a dark energy contribution which goes as $\left[r_{h} / a(t)\right]^{4}$, where $a$ is the brane scale factor and $r_{h}$ the black hole horizon size. The standard Hubble expansion law, $H^{2} \sim \rho / M_{p}^{2}$, prevails once the energy density on the brane is below the fundamental scale, which we assume to hold true always in our case including the inflaton energy density. In this case, $r_{h} \sim$ $V(\phi)^{-1 / 4}$, is much smaller than the scale factor after the inflaton decay, and therefore such a potential dark energy contribution, $\rho \sim\left(1 / a^{4}\right)$, gives a negligible contribution today.

Once the inflaton energy is redshifted into the bulk (from the brane point of view), then the challenge is to reproduce the standard hot big bang model, using only brane physics. In this regard our approach is simple: a real bang from the sneutrino cosmology.

The recent advancement made in neutrino experiments points towards the fact that the neutrinos have nonvanishing masses. The solar neutrino deficit is better understood if the electron neutrinos oscillate into the muon neutrinos controlled by the squared mass difference $\Delta m_{\text {solar }}^{2} \sim 7 \times$ $10^{-5} \mathrm{eV}^{2}$, with a large mixing angle, $\tan ^{2} \theta_{\text {solar }} \sim 0.5$ [16], whereas the atmospheric neutrino experiments indicate $\nu_{\mu}-\nu_{\tau}$ oscillations with $\Delta m_{\mathrm{atm}}^{2} \sim 2.5 \times 10^{-3} \mathrm{eV}^{2}$ and $\sin ^{2}(2 \theta) \simeq 1$ [17].

The above required masses are much smaller than those expected if the neutrinos were Dirac particles, since this would require a fine-tuning of the Dirac Yukawa couplings to one part in $10^{11}$, at least. The most natural explanation of such small masses, however, comes out if the neutrinos are Majorana particles. Then the small masses can be understood through the seesaw mechanism that involves large right handed neutrino masses [18]. One advantage of this mechanism is that the right handed neutrino mass breaks $L$ (or $B-L$ ) quantum number, which can be the origin of the observed baryon asymmetry. The conversion of the lepton asymmetry into the baryons via active SM sphalerons within a range of $10^{12} \geq T \geq 100 \mathrm{GeV}$ can help us produce the observed baryon asymmetry [19]. In a supersymmetric theory, a supersymmetric partner of the right handed neutrino, the sneutrino, induces leptogenesis, which in principle can be tested by the cosmic microwave background radiation through the baryon-isocurvature fluctuations; see, e.g., [20].

The next question is how to reheat and generate the adiabatic density perturbations. The right handed neutrinos naturally couple to the SM Higgs and the lepton doublets; therefore, they decay into the SM degrees of freedom. Indeed, as we promote our idea to supersymmetry, the sneutrino can decay into the Higgsinos and the sleptons. The lightest stable supersymmetric particle can easily account for the observed cold dark matter [21]. During inflation the sneutrino condensate obtains a nonvanishing vacuum expectation value (VEV), even though its energy density during inflation is subdominant, but after inflation, once the inflaton energy has been released away into the bulk, the sneutrino condensate decays and becomes the only source for the entropy production. Therefore, once inflation is over, the lightest sneutrino condensate, whose mass is lighter than the Hubble expansion rate during inflation, starts oscillating coherently and eventually decaying into the SM degrees of freedom [22].

Next comes the challenge for generating the adiabatic density perturbations, which happens naturally when the sneutrino condensate decays. During inflation the sneutrino condensate generates quantum fluctuations that are stretched outside the horizon. These perturbations are known as the isocurvature fluctuations; however, once inflation ends the dominant energy density is in the sneutrino condensate, which decays by converting its entire isocurvature perturbations to the adiabatic ones. This process is known as a curvaton scenario [23]; its supersymmetric implementation has been given in Ref. [24].

For the purpose of illustration, let us assume a simple superpotential for the sneutrino condensate

$$
W=(1 / 2) M_{N} \mathbf{N N}+h \mathbf{N L} \mathbf{H}_{\mathbf{u}},
$$

where $\mathbf{N}, \mathbf{L}$, and $\mathbf{H}_{\mathbf{u}}$ stand for the neutrino, the lepton, and the Higgs doublet. We have assigned an odd $R$-parity for the right handed sneutrinos. The only possible interactions with leptons and the Higgs fields are through the Yukawa matrix, $h_{i \ell}$, where $i=1,2,3$ and $\ell=e, \mu, \tau$, respectively. The superpotential term induces a potential, 
$V=M_{N}^{2} \tilde{N}^{2}$, where $\tilde{N}$ denotes the sneutrino. For simplicity we always assume a diagonal basis for the right handed neutrino mass matrix. The left handed neutrinos obtain masses via the seesaw mechanism, $m_{\nu} \sim$ $m_{D}^{\dagger} M_{N}^{-1} m_{D}$, where $m_{D}$ is the Dirac mass matrix.

The lightest right handed sneutrino, whose mass is smaller than the Hubble expansion rate during inflation, can act as a cosmic condensate [20]. The heavier sneutrinos roll to the bottom of the potential during inflation. They also generate isocurvature fluctuations, but their amplitude is suppressed compared to the lighter ones $[20,25]$. In this Letter we ignore their dynamics. We deal with only the lightest sneutrino, which we identify as the right handed electron sneutrino $\left(N_{1}\right)$ that we will just call hereafter $N$, and $M_{N}$ to its mass. We also assume that its largest Yukawa coupling is that of the $\tau$ doublet and that we take $h_{1 \tau} \sim 10^{-4}-10^{-5}$. Note that by taking $M_{N} \sim 10^{10} \mathrm{GeV}$, with $M_{N 2,3} \gg M_{N}$, we obtain a small electron-tau neutrino mixing in the left handed sector, as it seems to be required by the small $\theta_{13}$ angle in the neutrino experiments.

The important point is that the isocurvature perturbations seed the adiabatic ones on the largest scales [1]. Nevertheless, the amplitude of such fluctuations must be of the correct order of magnitude during inflation, which then requires

$$
V^{\prime \prime}\left(\tilde{N}_{*}\right)=M_{N}^{2} \sim \alpha^{2} H_{*}^{2}, \quad H_{*} / \tilde{N}_{*}=\delta,
$$

where the star denotes the value evaluated at the horizon crossing, $\alpha \ll 1$, and the perturbation, $\delta \sim 10^{-5}$. On the other hand, the scale of inflation can be parametrized by $V_{I}^{1 / 4} \sim\left(H_{*} M_{p}\right)^{1 / 2}$. The fluctuation in the sneutrino condensate can be imprinted upon radiation after its decay. In these terms, the spectral index of microwave temperature perturbations can then be evaluated as

$$
n_{s}-1=2 \dot{H}_{*} / H_{*}^{2}+(2 / 3)\left(M_{N} / H_{*}^{2}\right) \sim(2 / 3) \alpha^{2} .
$$

For $\alpha \sim 10^{-1}$, we find that $n_{s}$ is fairly close to 1 for $\dot{H}_{*} / H_{*}^{2} \approx 0$, which is consistent with the recent Wilkinson Microwave Anisotropy Probe observation of $n_{s}=0.99 \pm 0.04$ [26].

The perturbative decay rate of the lightest sneutrino condensate can be estimated by $\Gamma \sim\left|h_{1 \tau}\right|^{2} M_{N} / 4 \pi$, and the reheat temperature, $T_{r h} \sim 0.1 \sqrt{\Gamma M_{p}}$. We obtain

$$
T_{r h} \sim 10^{9}\left(h_{1 \tau} / 10^{-4}\right)\left(M_{N} / 10^{10}\right)^{1 / 2} \mathrm{GeV} .
$$

Note that the reheat temperature is low enough to avoid the gravitino problem (reheat temperature below $10^{9} \mathrm{GeV}$ ) [27]. The bound on the lightest right handed neutrino mass has to be around $10^{10} \mathrm{GeV}$, which sets the scale of inflation to be $H_{*} \sim 10^{10} / \alpha \mathrm{GeV}$. If $\alpha \sim 10^{-1}$, then $H_{*} \sim 10^{11} \mathrm{GeV}$ and $\tilde{N} \sim 10^{16} \mathrm{GeV}$, close to the grand unification scale. Note that we ignored the Dterm contributions in the sneutrino potential; however, it is safe to assume so if the sneutrino VEV is smaller than the scale at which the gauge group that embodies the right handed neutrino breaks down, e.g., the $\mathrm{SO}(10)$ gauge group. Note also that the right handed neutrino mass scale is different from the GUT scale, since it can actually come via some nonrenormalizable operators, as it happens in many $\mathrm{SO}(10)$ constructions.

Let us discuss baryogenesis. The sneutrino decay also induces lepton asymmetry due to the $C P$ violation. The $C P$ asymmetry in our scenario can be calculated by computing the interference between the tree-level and the one loop diagrams of $\tilde{N}$ going into $l_{\ell} \tilde{H}_{u}$ and the antislepton $l_{\ell}^{*} H_{u}^{*}$. The $C P$ asymmetry is given by $[19,28]$

$$
\epsilon \sim(\ln 2 / 8 \pi) \operatorname{Im} h_{3 \tau}^{* 2},
$$

where we assumed $h_{1 \tau} \geq h_{1 e}, h_{1 \mu}$, with $h_{3 \tau}$ dominance, and both $h_{1 \tau}$ and $M_{N}$ are real.

Now we can predict the overall light neutrino mass scale with the help of the seesaw formula, and we get $m_{\nu, \tau}=\left|h_{3 \tau}\right|^{2} \sin ^{2} \beta /\left(2 \sqrt{2} G_{F} M_{N, 3}\right)$, where $G_{F}$ is the Fermi constant, $M_{N, 3}$ is the mass of the right handed tau sneutrino, and $\tan \beta=\left\langle H_{u}\right\rangle /\left\langle H_{d}\right\rangle$. If we take $\left|h_{3 \tau}\right| \sim 10^{-2}$, with a typical $\tan \beta \sim 10$ and $M_{N, 3} \sim 10^{11} \mathrm{GeV}$ we obtain $m_{\nu, \tau} \sim 0.3 \mathrm{eV}$. Further note that this value is already at the desired scale for the right handed neutrino masses. Also a slightly smaller Yukawa coupling or a larger $M_{N 3}$ can easily bring the mass scale down without affecting other predictions.

The lepton asymmetry is converted by the SM sphalerons, and as a result the net baryon asymmetry is given by [28]

$$
n_{B} / s \sim \epsilon(8 / 15)\left(T_{r h} / M_{N}\right) .
$$

The ratio $T_{r h} / M_{N}$ arises due to the entropy dilution. We note that, with $\epsilon \sim 10^{-8}$, we can easily generate the baryon asymmetry of order one part in $10^{10}$ for $T_{r h} \sim$ $10^{9} \mathrm{GeV}$ and $M_{N} \sim 10^{10} \mathrm{GeV}$. The actual prediction is an interplay between $T_{r h}, M_{N}$, and $\epsilon$, but it is interesting to see that it is possible to obtain the right number without much fine-tuning.

Also note that the fluctuations in the reheat temperature lead to the fluctuations in the baryon asymmetry [20], $\delta\left(n_{B} / s\right) \propto \delta T_{r h}$; however, in our case it is possible to show that there are no baryon-isocurvature fluctuations generated due to the sneutrino decay. The fact that the sneutrino completely dominates the energy density while decaying, therefore converting all its isocurvature fluctuations into the adiabatic ones. In our simple setup we strictly predict $S_{B}=0$.

To summarize, in our setup, we have completely relaxed the inflaton sector, although inflation certainly solves the horizon and the flatness problems; however, the inflaton energy density need not reheat the Universe with the SM degrees of freedom. We showed that in a brane world setup it is possible that the inflaton energy density can be redshifted away into the bulk. The density 
perturbations, reheating the Universe with SM degrees of freedom, and the baryon asymmetry are all served by a single source, the right handed Majorana neutrino sector within supersymmetry. The neutrino sector can be embedded in a grand unified gauge group such as in $\mathrm{SO}(10)$. Interesting points to note are that in our case the sneutrino vev is consistent with the $\mathrm{SO}(10)$ breaking scale, the reheat temperature is low enough to avoid the gravitino problem, the spectral index is close to 1 , and there is no baryon-isocurvature fluctuations.

A. M. acknowledges Kari Enqvist, Jim Cline, Guy Moore, and Horace Stoica for helpful discussion.

[1] V.F. Mukhanov, H. A. Feldman, and R. H. Brandenberger, Phys. Rep. 215, 203 (1992).

[2] S. Sarkar, Rep. Prog. Phys. 59, 1493 (1996); K. A. Olive, G. Steigman, and T. P. Walker, Phys. Rep. 333, 389 (2000).

[3] D. H. Lyth and A. Riotto, Phys. Rep. 314, 1 (1998).

[4] R. Brandenberger, D. A. Easson, and A. Mazumdar, Phys. Rev. D 69, 083502 (2004).

[5] G. R. Dvali and S. H. Tye, Phys. Lett. B 450, 72 (1999); C. P. Burgess, M. Majumdar, D. Nolte, F. Quevedo, G. Rajesh, and R. J. Zhang, J. High Energy Phys. 0107 (2001) 047; J. Garcia-Bellido, R. Rabadan, and F. Zamora, J. High Energy Phys. 0201 (2002) 036; G. Shiu and S. H. Tye, Phys. Lett. B 516, 421 (2001); N. Jones, H. Stoica, and S. H. Tye, J. High Energy Phys. 0207 (2002) 051; K. Dasgupta, C. Herdeiro, S. Hirano, and R. Kallosh, Phys. Rev. D 65, 126002 (2002); B. s. Kyae and Q. Shafi, Phys. Lett. B 526, 379 (2002); S. Kachru, R. Kallosh, A. Linde, J. Maldacena, L. McAllister, and S. P. Trivedi, J. Cosmol. Astropart. Phys. 10 (2003) 013.

[6] A. Mazumdar, S. Panda, and A. Perez-Lorenzana, Nucl. Phys. B614, 101 (2001).

[7] L. Randall and R. Sundrum, Phys. Rev. Lett. 83, 4690 (1999).

[8] N. Arkani-Hamed, S. Dimopoulos, and G. Dvali, Phys. Lett. B 429, 263 (1998); Phys. Rev. D 59, 086004 (1999); I. Antoniadis, N. Arkani-Hamed, S. Dimopoulos, and G. Dvali, Phys. Lett. B 436, 257 (1998).

[9] V. A. Rubakov, Phys. Usp. 44, 871 (2001) [Usp. Fiz. Nauk 171, 913 (2001)].

[10] N. Kaloper and A. Linde, Phys. Rev. D 59, 101303 (1999); A. Mazumdar, Phys. Lett. B 469, 55 (1999); N. Arkani-Hamed et al., Nucl. Phys. B567, 189 (2000); R. N. Mohapatra, A. Pérez-Lorenzana, and C. A. de S. Pires, Phys. Rev. D 62, 105030 (2000); A. Mazumdar and A. Perez-Lorenzana, Phys. Lett. B 508, 340 (2001); A. M. Green and A. Mazumdar, Phys. Rev. D 65, 105022 (2002); A. Mazumdar, R. N. Mohapatra, and A. Pérez-Lorenzana, hep-ph/0310258.

[11] R. Maartens, D. Wands, B. A. Bassett, and I. Heard, Phys. Rev. D 62, 041301 (2000); A. Mazumdar and J. Wang,
Phys. Lett. B 490, 251 (2000); E. E. Flanagan, S. H. Tye, and I. Wasserman, Phys. Rev. D 62, 044039 (2000); A. Mazumdar, Phys. Rev. D 64, 027304 (2001); Nucl. Phys. B597, 561 (2001).

[12] F. Quevedo, Classical Quantum Gravity 19, 5721 (2002).

[13] P. Binetruy, C. Deffayet, and D. Langlois, Nucl. Phys. B565, 269 (2000); J. M. Cline, C. Grojean, and G. Servant, Phys. Rev. Lett. 83, 4245 (1999).

[14] S. L. Dubovsky, V. A. Rubakov, and P. G. Tinyakov, Phys. Rev. D 62, 105011 (2000).

[15] A. Hebecker and J. March-Russell, Nucl. Phys. B608, 375 (2001).

[16] Super-Kamiokanade Collaboration, S. Fukuda et al., Phys. Rev. Lett. 86, 5656 (2001); SNO Collaboration, Q. R. Ahmad et al., Phys. Rev. Lett. 87, 071301 (2001).

[17] Super-Kamiokanade Collaboration, S. Fukuda et al., Phys. Rev. Lett. 85, 3999 (2000).

[18] M. Gell-Mann, P. Ramond, and R. Slansky, in Supergravity, edited by $\mathrm{P}$. van Niewenhuizen and D. Z. Freedman (North-Holland, Amsterdam, 1979); T. Yanagida, Proceedings of the Workshop on Unified Theory and Baryon Number in the Universe, edited by O. Sawada and A. Sugamoto (KEK, Ibaraki, Japan, 1979); R. N. Mohapatra and G. Senjanović, Phys. Rev. Lett. 44, 912 (1980).

[19] M. Fukugita and T. Yanagida, Phys. Lett. B 174, 45 (1986); Phys. Rev. D 42, 1285 (1990).

[20] A. Mazumdar, Phys. Rev. Lett. 92, 241301 (2004); Phys. Lett. B 580, 7 (2004).

[21] J. R. Ellis et al., Phys. Lett. B 565, 176 (2003).

[22] M. Postma and A. Mazumdar, J. Cosmol. Astropart. Phys. 0401 (2004) 005.

[23] K. Enqvist and M. S. Sloth, Nucl. Phys. B626, 395 (2002); D. H. Lyth and D. Wands, Phys. Lett. B 524, 5 (2002); T. Moroi and T. Takahashi, Phys. Lett. B 522, 215 (2001); 539, 303(E) (2002)].

[24] K. Enqvist, S. Kasuya, and A. Mazumdar, Phys. Rev. Lett. 90, 091302 (2003); K. Enqvist, A. Jokinen, S. Kasuya, and A. Mazumdar, Phys. Rev. D 68, 103507 (2003); M. Postma, Phys. Rev. D 67, 063518 (2003); K. Hamaguchi et al., Phys. Rev. D 69, 063504 (2004); K. Hamaguchi et al., Phys. Rev. D 65, 043512 (2002); J. McDonald, Phys. Rev. D 68, 043505 (2003); K. Enqvist, S. Kasuya, and A. Mazumdar, hep-ph/ 0311224; K. Enqvist, A. Mazumdar, and A. PerezLorenzana, hep-th/0403044.

[25] A. D. Linde and V. F. Mukhanov, Phys. Rev. D 56, 535 (1997); A. R. Liddle and A. Mazumdar, Phys. Rev. D 61, 123507 (2000).

[26] D. N. Spergel et al., Astrophys. J. Suppl. Ser. 148, 175 (2003).

[27] J. Ellis et al., Phys. Lett. B 145, 181 (1984); A. L. Maroto and A. Mazumdar, Phys. Rev. Lett. 84, 1655 (2000); R. Kallosh et al., Phys. Rev. D 61, 103503 (2000); R. Allahverdi et al., Phys. Rev. D 64, 023516 (2001).

[28] H. Murayama, H. Suzuki, T. Yanagida, and J. Yokoyama, Phys. Rev. Lett. 70, 1912 (1993). 